\title{
Numerical and Experimental Analyses of Vibrations of Annular Plates with Multiple Eccentric Holes
}

\author{
S. Noga \\ Rzeszów University of Technology, Rzeszów, Poland \\ noga@prz.edu.pl
}

This paper deals with the free transverse vibrations of annular plates with geometrical discontinuities in form of eccentric holes and rims. The finite element method and experimental studies are used. Solid and shell finite element models are employed in the numerical analysis using the commercial ANSYS software. The calculated results are verified by the original experimental data via innovative laser measurement techniques. Numerical and experimental results of this study adequately describe the phenomenon of dividing natural frequency values corresponding to the mode shapes, where the number of nodal diameters is a factor of the number of through holes. The proposed procedure of analysis of transverse vibrations of annular plates with eccentric through holes arranged over the specified radius is recommended for engineering applications.

Keywords: cyclic symmetry modeling, transverse vibration, distorted mode shapes, FEM.

Introduction. The problem of free transverse vibrations of circular and annular plates with geometrical discontinuities is of theoretical and practical importance, insofar as such systems have many engineering applications in the railway and aviation industries [1-3]. The fundamental vibration theory of annular and circular plate systems are presented in monographs [4, 5]. An exact solution for the problem of vibration of a circular plate weakened along an internal concentric circle and simply supported along its edge has been introduced in study [6]. Work [2] deals with analysis of vibration problem of annular plates with different types of geometrical discontinuities (holes, slots, cutouts, etc.). To solve the transverse vibration problem of the above systems the semi-analytical Ritz method is employed. Analytical formulation of the vibration problems of plates with eccentric holes is discussed in papers [7, 8]. In article [7], solution of this problem is achieved by using the finite element method (FEM), while authors [8] apply the indirect boundary element method to solve similar problems. Researchers [9] proposed a new type of finite elements designed to solve the free transverse vibration problem of annular plates with periodic radial through cracks. In works $[1,3,10,11]$, the finite element (FE) technique is used to analyze the free transverse vibrations of annular plates with eccentric holes arranged periodically over specified radius, while the problem of shape distortion of normal modes of studied systems is discussed and attempts to derive the algorithm to identify the proper distorted mode shapes are made. In article [12], the FEM technique and fatigue test analysis are employed to determine the influence of inelastic resistance on vibrodiagnostic parameters indicating the presence of some types of damages. Due to the possibility of reducing the size of FE models, the cyclic symmetry modeling technique becomes quite a lucrative approach in vibration analysis of circular and annular systems [3, 4]. In paper [13], cyclic symmetry features are used in vibration analysis of periodic stators of permanent magnet motors. In work [14], a similar technique is employed to solve vibration problem of circular systems with different types of damages. In both above papers, the analytical, numerical, and experimental studies are conducted. In article [15], the cyclic symmetry modeling is included in the solving process of transverse vibration problems of representative compound annular plate. A more complementary discussion on transverse vibrations of toothed gears treated as annular plates with a complex geometry and including 
the cyclic symmetry technique is presented in monograph [3], which deals with free transverse vibrations of annular plates with geometrical discontinuities in form of eccentric holes arranged periodically over specified radius and rim. FEM analysis (including the cyclic symmetry model) and experimental studies are conducted. Numerical and experimental results discussed in this paper confirm the existence of the phenomenon of dividing natural frequency values corresponding to the mode shapes, where number of nodal diameters is a factor of the number of through holes. This paper continues recent author investigations concerning the transverse vibrations of complex systems [16].

1. Problem Formulation. As was follows from the introduction, circular or annular plate type systems with diverse cases of geometric discontinuities, (e.g., eccentric holes and slots, protrusions in the form of annuli, hubs, etc.) are used during vibration modeling and analysis of such mechanical systems as toothed wheels, railway wheels, aircraft engine turbines, etc. The main aim of this paper is to develop reliable engineering procedures for analyzing transverse vibrations of annular plates with geometric discontinuities such as eccentric through holes arranged along the specified radius. Figure 1 depicts the geometrical model assumed for the considered system.
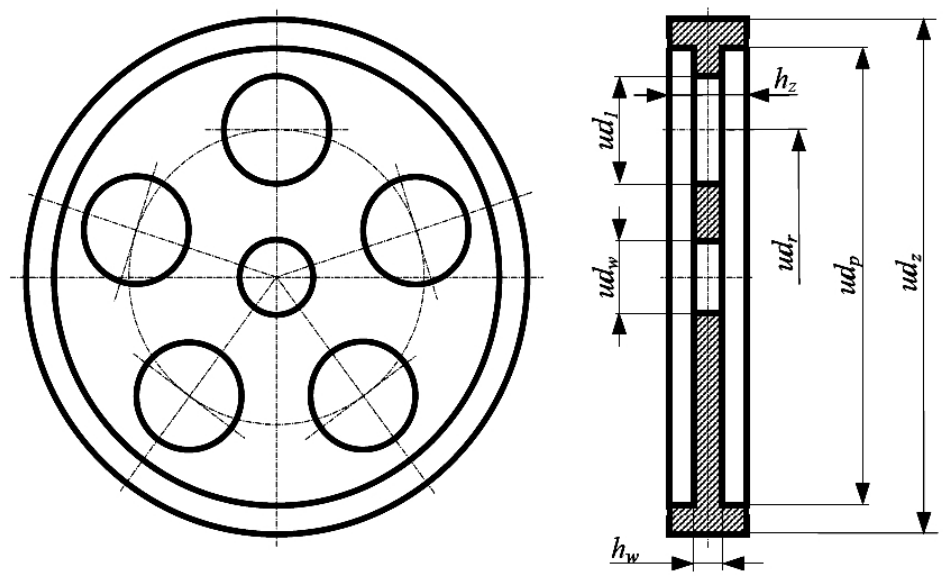

Fig. 1. Geometry of the system under study.

Basic geometric dimensions (diameter and thickness values) and technical data of the system under consideration are specified in Table 1 . The model is composed of an annular plate with external rim and five eccentric holes arranged along a given radius. The overall arrangement of the system under study complies with commonly used solutions applied to toothed wheels of aviation gear boxes, where through holes are made in the wheel disk, in order to decrease the weight.

$\mathrm{T}$ a b 1 e 1

Parameters of the System under Study

\begin{tabular}{|c|c|c|c|c|c|c|c|c|c||}
\hline $\begin{array}{c}d_{z}, \\
\mathrm{~mm}\end{array}$ & $\begin{array}{c}d_{p}, \\
\mathrm{~mm}\end{array}$ & $\begin{array}{c}d_{r}, \\
\mathrm{~mm}\end{array}$ & $\begin{array}{c}d_{w}, \\
\mathrm{~mm}\end{array}$ & $\begin{array}{c}d_{1}, \\
\mathrm{~mm}\end{array}$ & $\begin{array}{c}h_{z}, \\
\mathrm{~mm}\end{array}$ & $\begin{array}{c}h_{w}, \\
\mathrm{~mm}\end{array}$ & $v$ & $\begin{array}{c}E, \\
\mathrm{MPa}\end{array}$ & $\begin{array}{c}\rho, \\
\mathrm{kg} / \mathrm{m}^{3}\end{array}$ \\
\hline 110.09 & 98.0 & 62.7 & 15.2 & 22.4 & 7.0 & 2.29 & 0.28 & $2 \cdot 10^{5}$ & $7.85 \cdot 10^{3}$ \\
\hline
\end{tabular}

The commercial ANSYS package is used for the FEM analysis. The dynamic analysis, which includes determination of values of vibration natural frequencies, is a critical step made in the structural design process (particularly, in the aircraft industry). 
Each particular natural frequency of vibrations in the engineering design routine is identified based on the shape of the respective normal modes. Although for the case of a solid plate, solving of such problem poses no difficulty, in the systems with an irregular geometry (e.g., system shown in Fig. 1), these forms are distorted as compared to the respective system with a regular geometry. An algorithm, which allows one to determine the correspondence between mode shapes of a solid plate and a plate with eccentric through holes, is discussed in detail elsewhere [1, 10, 11]. From the engineering application standpoint, this algorithm can be a good alternative to the solutions proposed in $[2,8]$. One of the main aims of this work is the experimental verification of the numerical simulation results. According to the classical theory of transverse vibrations of circular and annular solid plates, for each solution where nodal lines form nodal diameters, two identical systems of nodal lines rotated with reference to one another by the angle $\alpha$ are obtained [3-5]:

$$
\alpha=\frac{\pi}{2 n}
$$

where $n$ is the number of nodal diameters. In compliance with conventional terminology of the theory of plates with a circular symmetry [5], each natural frequency is denoted by $\omega_{m n}$, where $m$ refers to the number of nodal circles. The fact that through holes would deform nodal lines of the mode shapes of transverse vibrations is quite expected. Additionally, at some frequencies, where there are double solutions, two different values are obtained with two corresponding normal modes of different shapes [1-3, 8]. This applies to the solutions where the nodal lines form nodal diameters. For the case under study, the values are likely to be separated for the frequencies of $\omega_{15}$ and $\omega_{25}$, which issue will be discussed below. The quality of the developed numerical models is determined by computing the relative error for each frequency defined via Eq.(2) [3, 4]:

$$
\varepsilon=\frac{\omega^{f}-\omega^{e}}{\omega^{e}} \cdot 100 \%,
$$

where $\omega^{f}$ is the natural frequency from the numerical model and $\omega^{e}$ is the natural frequency of the actual system. The parameter $\varepsilon$ in Eq. (2) is referred to in [3] as the frequency error.

2. FE Representations. In elaboration of the FE models of the system under study, the cyclic symmetry feature of the system is taken into account, which simplifies both the modeling process, and analysis of the system vibrations. The main advantage of this feature is in its application in vibration analysis of the representative segment, which is a portion of the system with a cyclic symmetry under consideration. Such an approach significantly reduces the size of the FE models. The effectiveness of models of this property, as compared to complete models, is more extensively discussed in papers $[3,13-15]$. In the case under consideration, a segment including one fifth part of the model of the system shown in Fig. 1 is used (see Fig. 2a).

The specifications of the system under study are shown in Table 1. Two FE models of the plate are applied: with solid and shell elements. In the first case, a FE solid model is prepared with the use of tetrahedral solid element (solid187), with ten nodes of three degrees of freedom in each node. In the second case, a FE model with shell elements (shell281) with eight nodes and with six degrees of freedom in each node, is used. In the meshing process of each particular model, the standard ANSYS package procedures are used. For the first model, it is assumed that the edge length of an element does not exceed $2.3 \mathrm{~mm}$, which is a value equal to the thickness of the plate thinner part (see Table 1). This 


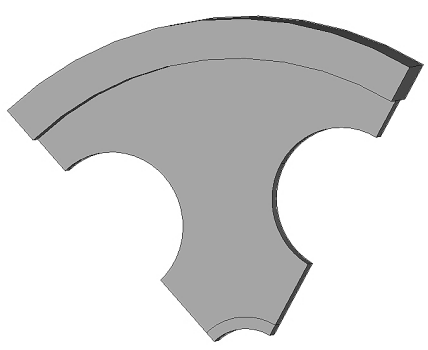

a

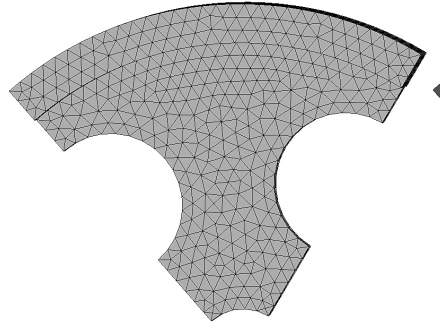

b

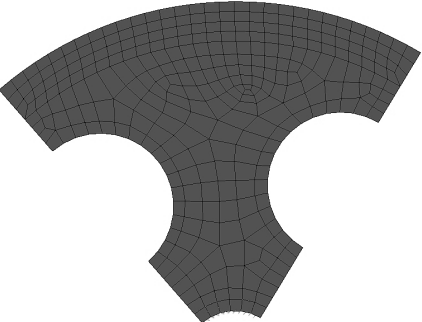

C

Fig. 2. The cyclic symmetry model (a), first FE model (b), and second FE model (c).

model is composed of 3461 solid elements. For the second FE model, it is assumed that the edge length of the element located in periphery of the solid one is approximately equal to $2 \mathrm{~mm}$. The second FE model contains 414 surface elements. Both models are depicted in Fig. $2 b$, c.

Two kinds of boundary conditions can be distinguished in the elaborated models: the first one represents the cyclic symmetry of the model and is imposed by the system (i.e., automatically by respective procedures within the ANSYS package), while the second one describes a prospective attachment of the system for the measuring experiment. In the latter cases, the boundary conditions that consider this attachment are imposed on the respective nodes. The degrees of freedom associated with node displacement along the axial symmetry axis are excluded for the nodes located along the surface and labeled as "fixing" in each model in Fig. 3. It is noteworthy that the number of elements in the solid FE model is more than eight times higher than that in the shell one.

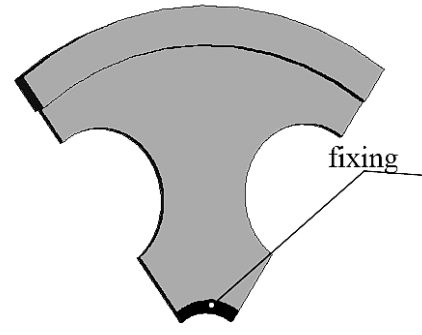

a

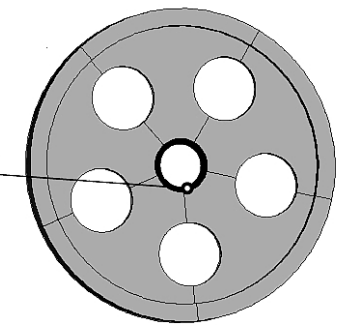

b

Fig. 3. Boundary conditions for the cyclic symmetry (a) and cyclic expansion (b) models.

3. Experimental. The experimental verification of the proposed FEM models for the system, as well as the methodology used for identifying the mode shapes of transverse vibrations of the plate distorted by holes, discussed below have been briefly discussed in [16]. The Polytec measuring system used in this study (Fig. 4) is composed of PSV-400 laser vibrometer, Polytec DMS DATA MANAGEMENT SYSTEM, NOLIAC piezoelectric sensors of CMAP03, CMAP06, EPA-104 piezoelectric sensor amplifier, TIRAvib5200 electrodynamic exciter, and a Textronic TGA12101 type signal generator.

The measuring experiment is planned and performed in such a way as to identify the natural forms in the system under consideration. For the measuring tests, four objects of the geometric characteristics (as shown in Fig. 1) and diameter values of $d_{1}$ of the through holes described in Table 2 have been produced, whose geometrical dimensions and material properties are tabulated in Table 1. 
T a b 1 e 2

\section{Diameters of Holes in Objects under Study}

\begin{tabular}{|c|c|c|c|c||}
\hline \hline \multirow{2}{*}{$\begin{array}{c}d_{1}, \\
\mathrm{~mm}\end{array}$} & 1 & 2 & 3 & 4 \\
\cline { 2 - 5 } & 0 & 7.28 & 15.68 & 22.40 \\
\hline
\end{tabular}

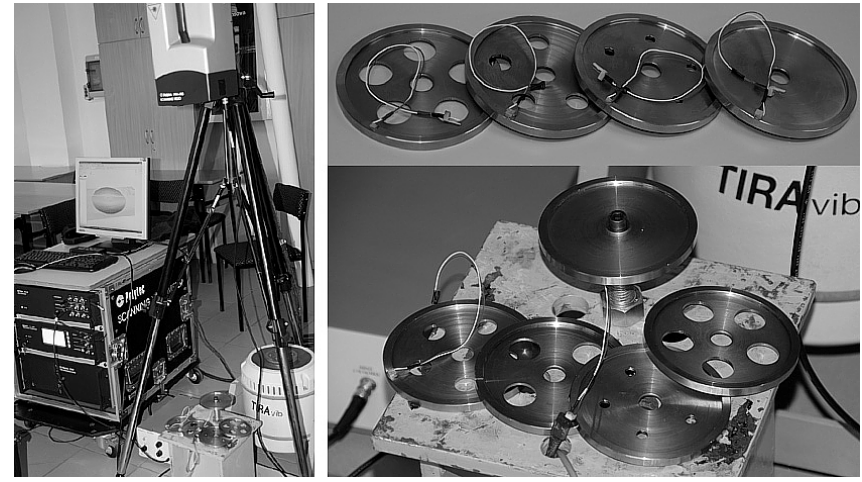

Fig. 4. The measuring test.

These objects are excited by a harmonic signal and then by a pseudo random noise at $20-30 \mathrm{kHz}$ band. A mesh of measuring points is superimposed on each object. The number of selected measuring points for each object was 500 to 1000 . At each point the response of the system is measured along the transverse direction with a laser vibrometer. The values of excited and identified natural frequencies of transverse vibrations in the objects under consideration, are given in Tables 3-6. When identifying the natural forms for the plates with through holes, the results obtained from FE models of the objects were used for getting more insight on the shape distortion induced by the through holes. For frequencies of plates with through holes $\omega_{25}$ and $\omega_{15}$, two normal modes of different shapes and corresponding frequencies are identified. Such findings corroborate earlier findings of [2] regarding separation of natural frequency values related to the forms, where the number of nodal diameters is a factor of the number of through holes.

$\mathrm{T}$ a b 1 e 3

Experimental Natural Frequencies $\omega_{m n}(\mathbf{H z})$ for the Solid Plate

\begin{tabular}{||c|c|c|c|c|c|c|c|c|c||}
\hline \hline \multirow{2}{*}{$m$} & \multicolumn{9}{|c||}{$n$} \\
\cline { 2 - 10 } & 0 & 1 & 2 & 3 & 4 & 5 & 6 & 7 & 8 \\
\hline 1 & 824.2 & 515.6 & 1456.3 & 4057.0 & 7704.7 & $12,231.3$ & $17,532.8$ & $23,437.5$ & $29,867.2$ \\
\hline 2 & 5146.9 & 5878.1 & 7637.5 & $10,921.9$ & $15,026.6$ & $19,984.4$ & $25,410.2$ & - & - \\
\hline 3 & $13,736.0$ & $14,132.8$ & $17,828.1$ & $21,894.5$ & $27,578.0$ & - & - & - & - \\
\hline \hline
\end{tabular}

$\mathrm{T}$ a b 1 e 4

Experimental Natural Frequencies $\omega_{m n}(\mathbf{H z})$ for the Plate with Holes of $\boldsymbol{d}_{1}=7.28 \mathrm{~mm}$

\begin{tabular}{||c|c|c|c|c|c|c|c|c|c||}
\hline \hline \multirow{2}{*}{$m$} & \multicolumn{9}{|c||}{$n$} \\
\cline { 2 - 10 } & 0 & 1 & 2 & 3 & 4 & 5 & 6 & 7 & 8 \\
\hline 1 & 780.5 & 443.8 & 1441.4 & 4014.5 & 7632.8 & $12,125.0$ & $17,328.1$ & $23,097.7$ & $29,289.1$ \\
\hline 2 & 4890.6 & 5339.8 & 7253.9 & $10,425.0$ & $14,441.4$ & $18,871.1$ & $24,171.9$ & - & - \\
& & & & & & $19,246.1$ & & & - \\
\hline 3 & $13,052.0$ & $13,412.5$ & $16,386.7$ & $20,722.7$ & $26,191.4$ & - & - & - & - \\
\hline \hline
\end{tabular}


Numerical and Experimental Analyses of Vibrations...

T a b 1 e 5

Experimental Natural Frequencies $\omega_{m n}(\mathrm{~Hz})$ for the Plate with Holes of $\boldsymbol{d}_{\mathbf{1}}=\mathbf{1 5 . 6 8} \mathrm{mm}$

\begin{tabular}{||c|c|c|c|c|c|c|c|c|c||}
\hline \multirow{2}{*}{$m$} & \multicolumn{9}{|c||}{$n$} \\
\cline { 2 - 10 } & 0 & 1 & 2 & 3 & 4 & 5 & 6 & 7 & 8 \\
\hline 1 & 740.6 & 490.6 & 1425.4 & 4000.8 & 7581.3 & $12,078.1$ & $17,183.6$ & $22,695.3$ & $28,023.4$ \\
\hline 2 & 4859.4 & 5618.8 & 7074.2 & $10,489.1$ & $14,032.8$ & $16,859.4$ & $23,050.7$ & - & - \\
\hline 3 & & & & & & $20,843.6$ & & & - \\
\hline
\end{tabular}

T a b 1 e 6

Experimental Natural Frequencies $\omega_{m n}(\mathrm{~Hz})$ the Plate with Holes of $d_{1}=22.4 \mathrm{~mm}$

\begin{tabular}{|c|c|c|c|c|c|c|c|c|c||}
\hline \multirow{2}{*}{$m$} & \multicolumn{7}{|c||}{$n$} \\
\cline { 2 - 10 } & 0 & 1 & 2 & 3 & 4 & 5 & 6 & 7 & 8 \\
\hline 1 & 716.8 & 409.4 & 1424.2 & 4000.4 & 7642.2 & $\begin{array}{l}11,885.9 \\
12,265.6\end{array}$ & $17,480.0$ & $22,988.3$ & $29,117.2$ \\
\hline 2 & 5276.6 & 5868.8 & 7278.1 & $12,460.9$ & $15,085.9$ & $\begin{array}{l}17,707.0 \\
21,703.1\end{array}$ & $22,914.1$ & - & - \\
\hline 3 & $11,923.0$ & $12,565.6$ & $15,134.4$ & $19,410.2$ & $24,898.4$ & - & - & - & - \\
\hline
\end{tabular}

4. Numerical Analysis. Calculations are made for the above FE models and the natural frequencies are determined, as well as the corresponding mode shapes of transverse vibrations. For each object under study the FE models are elaborated according with the rules discussed in section two. The analysis is performed in a wide range of frequencies where the upper limit corresponds to the frequency $\omega_{18}$ associated with eight nodal diameters of flexural form. For the identification of some natural forms of vibrations it was necessary to apply the algorithm to identify the proper distorted mode shapes which was discussed in works $[1,3,10,11]$. Results of FEM analysis (normal modes) are compared with the experimental ones. Similar to the previous case, both FE models of the plates with holes exhibit two different values of frequency of $\omega_{25}$ (and for $\omega_{15}$ frequency of plate with through holes of $d_{1}=22.4 \mathrm{~mm}$ ). Different mode shapes correspond to each value. For brevity sake, Tables 7-12 show only the frequency error determined for FE models (in cases of a solid plate and plate with through holes of $d_{1}=22.4 \mathrm{~mm}$ for two FE models and, in other cases, only for FE shell models).

T a b 1 e 7

Frequency Errors $\varepsilon_{m n}(\%)$ the FE Solid Model Numerical Solution in Comparison to the Experimental Data

\begin{tabular}{||c|c|c|c|c|c|c|c|c|c||}
\hline \multirow{2}{*}{$m$} & \multicolumn{9}{|c||}{$n$} \\
\cline { 2 - 10 } & 0 & 1 & 2 & 3 & 4 & 5 & 6 & 7 & 8 \\
\hline 1 & -0.33 & 13.77 & 4.40 & 1.28 & 0.72 & 0.53 & 0.24 & 0.11 & -0.10 \\
\hline 2 & 5.80 & 2.01 & 1.85 & -0.47 & -0.38 & -1.14 & -1.17 & - & - \\
\hline 3 & 4.29 & 7.68 & 0.24 & 1.14 & 0.56 & - & - & - & - \\
\hline
\end{tabular}

The best results are obtained via the FE models of the solid plate: in both cases, similar error values for each particular frequency are observed. The shell FE model is somewhat more favorable, insofar as only in 8 cases out of 21 the frequency errors are higher than those of the solid FE model. The results of FEM modeling of the solid plate can 
T a b 1 e 8

Frequency Errors $\varepsilon_{m n}(\%)$ of the FE Shell Model Numerical Solution in Comparison with the Experimental Data for the Solid Plate

\begin{tabular}{||c|c|c|c|c|c|c|c|c|c||}
\hline \multirow{2}{*}{$m$} & \multicolumn{9}{|c||}{$n$} \\
\cline { 2 - 10 } & 0 & 1 & 2 & 3 & 4 & 5 & 6 & 7 & 8 \\
\hline 1 & -0.37 & 13.23 & 4.24 & 1.19 & 0.60 & 0.36 & -0.01 & -0.23 & -0.55 \\
\hline 2 & 5.40 & 1.62 & 1.61 & -0.55 & -0.46 & -1.29 & -1.45 & - & - \\
\hline 3 & 4.00 & 7.19 & -0.43 & 0.38 & -0.27 & - & - & - & - \\
\hline
\end{tabular}

T a b 1 e 9

Frequency Errors $\varepsilon_{m n}(\%)$ of the FE Shell Model Numerical Solution in Comparison with the Experimental Data for the Solid Plate with Holes of $d_{1}=7.28 \mathrm{~mm}$

\begin{tabular}{||c|c|c|c|c|c|c|c|c|c||}
\hline \hline \multirow{2}{*}{$m$} & \multicolumn{9}{|c||}{$n$} \\
\cline { 2 - 10 } & 0 & 1 & 2 & 3 & 4 & 5 & 6 & 7 & 8 \\
\hline 1 & 4.60 & 30.60 & 5.06 & 2.09 & 1.36 & 0.97 & 0.87 & 0.82 & 0.73 \\
\hline 2 & 10.12 & 11.02 & 6.04 & 3.23 & 2.55 & 2.43 & 2.2 & - & - \\
\hline 3 & 8.42 & 11.73 & 6.76 & 4.72 & 3.02 & - & - & - & - \\
\hline
\end{tabular}

T a b 1 e 10

Frequency Errors $\varepsilon_{m n}(\%)$ of the FE Shell Model Numerical Solution in Comparison with the Experimental Data for the Solid Plate with Holes of $d_{1}=15.68 \mathrm{~mm}$

\begin{tabular}{||c|c|c|c|c|c|c|c|c|c||}
\hline \hline \multirow{2}{*}{$m$} & \multicolumn{9}{|c||}{$n$} \\
\cline { 2 - 10 } & 0 & 1 & 2 & 3 & 4 & 5 & 6 & 7 & 8 \\
\hline 1 & 6.78 & 13.84 & 5.58 & 2.13 & 1.84 & 1.43 & 1.80 & 1.74 & 2.75 \\
\hline 2 & 13.16 & 7.71 & 9.35 & 5.87 & 5.08 & 4.50 & 5.48 & - & - \\
& & & & & & 2.97 & & & - \\
\hline 3 & 12.54 & 14.90 & 9.43 & 6.65 & 6.00 & - & - & - & - \\
\hline
\end{tabular}

$\mathrm{T}$ a b 1 e 11

Frequency Errors $\varepsilon_{m n}(\%)$ of the FE Solid Model Numerical Solution in Comparison with the Experimental Data for the Solid Plate with Holes of $d_{1}=22.4 \mathrm{~mm}$

\begin{tabular}{||c|c|c|c|c|c|c|c|c|c||}
\hline \hline \multirow{2}{*}{$m$} & \multicolumn{9}{|c||}{$n$} \\
\cline { 2 - 10 } & 0 & 1 & 2 & 3 & 4 & 5 & 6 & 7 & 8 \\
\hline 1 & 3.61 & 26.92 & 5.16 & 2.23 & 2.15 & $\begin{array}{l}1.43 \\
1.00\end{array}$ & 2.42 & 1.56 & 1.88 \\
\hline 2 & 10.29 & 8.52 & 6.52 & 4.39 & 4.04 & $\begin{array}{l}2.24 \\
3.62\end{array}$ & 7.55 & - & - \\
\hline 3 & 10.84 & 10.52 & 8.81 & 6.52 & 5.24 & - & - & - & - \\
\hline
\end{tabular}

be considered as satisfactory. In case of plates with holes, results are less satisfactory as compared to those discussed earlier, although similar error values that refer to each particular frequency, one can observe in both cases of FE models (Tables 9-12). Shell models of the considered systems seem to be more promising. Figures 5-7 show the 
T a b 1 e 12

Frequency Errors $\varepsilon_{m n}(\%)$ the FE Shell Model Numerical Solution in Comparison to the Experimental Data for the Plate with Holes of $d_{1}=22.4 \mathrm{~mm}$

\begin{tabular}{|c|c|c|c|c|c|c|c|c|c|}
\hline \multirow[t]{2}{*}{$m$} & \multicolumn{9}{|c|}{$n$} \\
\hline & 0 & 1 & 2 & 3 & 4 & 5 & 6 & 7 & 8 \\
\hline 1 & 3.43 & 26.23 & 4.96 & 2.12 & 2.01 & $\begin{array}{l}1.27 \\
0.83\end{array}$ & 2.16 & 1.21 & 1.47 \\
\hline 2 & 9.88 & 8.05 & 6.11 & 4.25 & 3.81 & $\begin{array}{l}2.06 \\
3.36\end{array}$ & 7.20 & - & - \\
\hline 3 & 10.08 & 9.69 & 7.71 & 5.43 & 4.15 & - & - & - & - \\
\hline
\end{tabular}

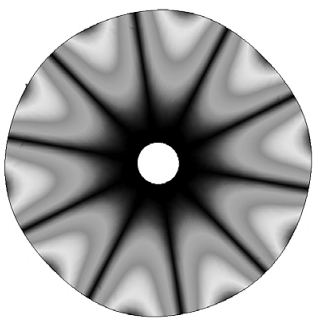

a

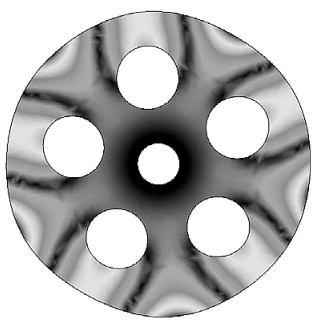

b

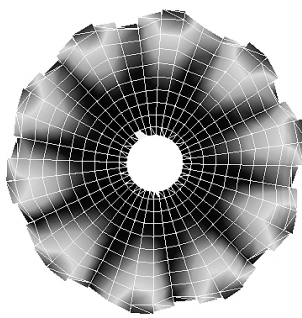

c

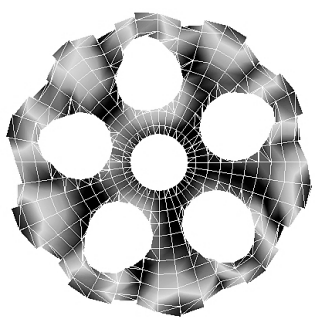

d

Fig. 5. Mode shapes related to frequency $\omega_{15}$ : (a), (b) FE shell model; (c), (d) experimental results.

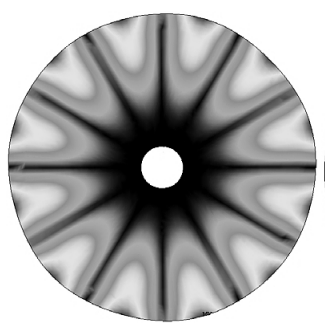

a

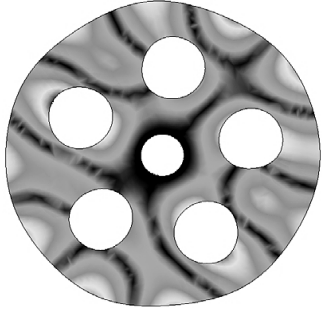

b

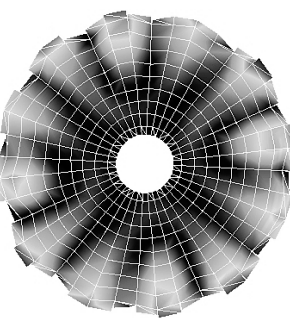

$\mathrm{c}$

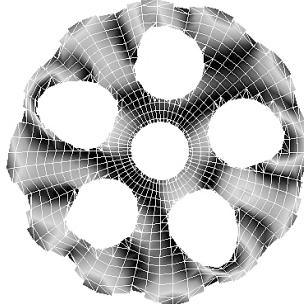

d

Fig. 6. Mode shapes related to frequency $\omega_{16}$ : (a), (b) FE shell model; (c), (d) experimental results.

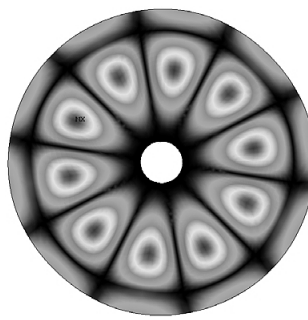

a

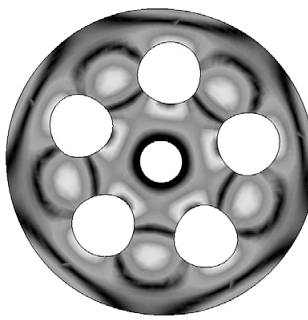

b

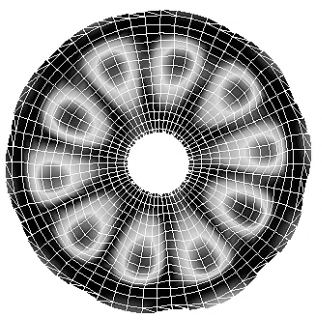

C

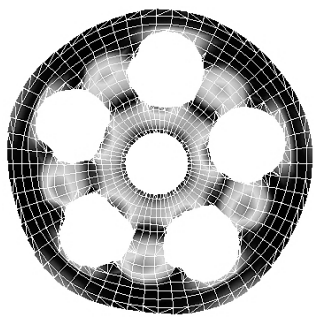

d

Fig. 7. Mode shapes related to frequency $\omega_{25}:(a),(b)$ FE shell model; (c), (d) experimental results.

selected natural forms of transverse vibrations obtained from the objects under study and corresponding FE shell models. The experimental forms are not so regular as those predicted by the FEM analysis (Figs. 5-7), but still easily identifiable.

The obtained numerical and experimental results can be discussed from the standpoint of the method of determining the correspondence between mode shapes of a solid plate and 


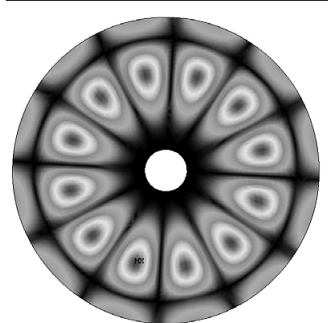

a

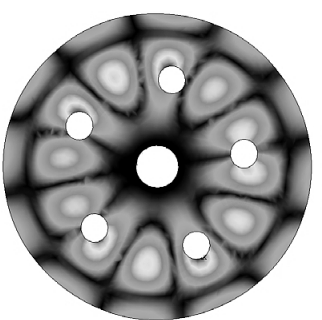

$\mathrm{b}$

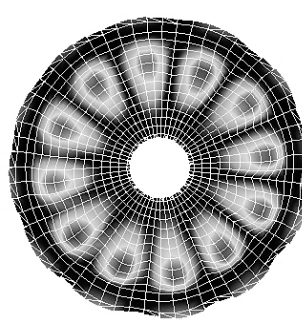

$\mathrm{c}$

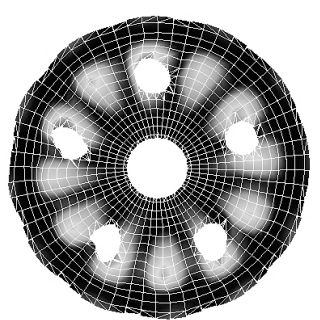

d

Fig. 8. Mode shapes related to frequency $\omega_{26}$ : (a), (b) FE shell model; (c), (d) experimental results $\left[(\mathrm{a}, \mathrm{c}) d_{1}=0 ;(\mathrm{b}, \mathrm{d}) d_{1}=7.28 \mathrm{~mm}\right]$.

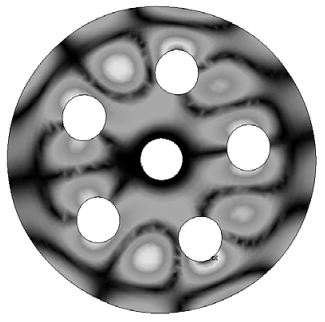

a

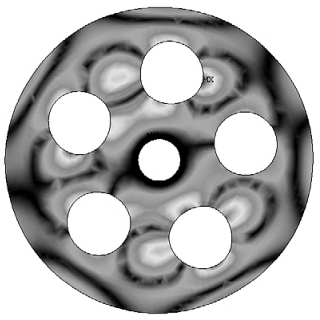

b

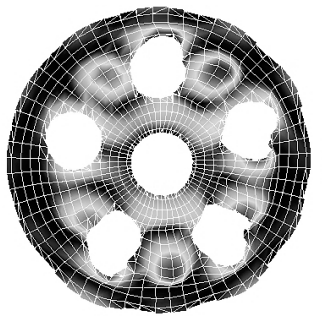

C

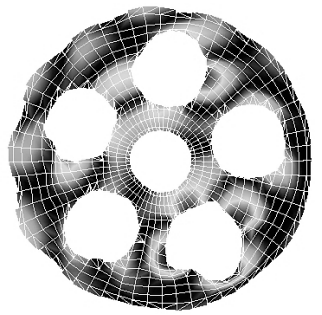

d

Fig. 9. Mode shapes related to frequency $\omega_{26}$ : (a), (b) FE shell model; (c), (d) experimental results $\left[(\mathrm{a}, \mathrm{c}) d_{1}=15.68 \mathrm{~mm} ;(\mathrm{b}, \mathrm{d}) d_{1}=22.4 \mathrm{~mm}\right]$.

a plate with eccentric through holes studies proposed in $[1,3,11]$. Consider the normal modes corresponding to natural frequency of $\omega_{26}$. Figures 8 and 9 depict the calculated and experimental mode shapes of four objects with diameter $d_{1}$ of the through holes given in Table 2, which indicate that through holes generate the distortion of nodal lines of the mode shapes. As in the above cases, a similarity of shapes of the corresponding natural forms achieved from the FEM solutions and experiment is evident. Both the FEM solutions and test results allow for tracing distortions of node lines associated with the nodal diameters and nodal circles due to availability through holes. It is noteworthy that these studies cover a wide range of frequencies $(400-30,000 \mathrm{~Hz})$.

Conclusions. In this paper, problems of transverse vibrations of plates with a circular symmetry and irregular geometry are discussed based on the original FEM analysis and experimental studies of annular plates with such geometrical discontinuities as through holes and thickened periphery. Solid and shell FE models are used for numerical simulation of this problem. More promising results with lower frequency errors and model sizes are obtained in case of application of the shell FEM models. The simulation and experimental results obtained confirm the phenomenon of dividing natural frequency values corresponding to the normal modes, where the number of nodal diameters is a factor of the number of through holes. From the engineering standpoint, the proposed method of vibration analysis of circular symmetric systems with a disturbed geometry, is a good alternative to the available numerical solutions, since it can be based on application of the standard ANSYS commercial package for the analysis.

1. R. Bogacz and S. Noga, "Free transverse vibration analysis of a toothed gear," Arch. Appl. Mech., 82, No. 9, 1159-1168 (2012).

2. H. Vinayak and R. Singh, "Eigensolutions of annular-like elastic disks with intentionally removed or added material," J. Sound Vib., 192, No. 4, 741-769 (1996). 
3. S. Noga, Analytical and Numerical Problems of Systems with Circular Symmetry Vibrations [in Polish], Publishing House of Rzeszów University of Technology, Rzeszów (2015).

4. C. de Silva, Vibration and Shock Handbook, CRC Press, Taylor \& Francis, Boca Raton, FL (2005).

5. A. W. Leissa, Vibration of Plates, NASA SP 160, US Government Printing Office, (1969).

6. L. Bhaskara Rao and C. Kameswara Rao, "Analysis of vibration natural frequencies of rotationally restrained and simply supported circular plate with weakened interior circle due to an angular crack," Strength Mater., 47, No. 6, 859-869 (2015).

7. L. Cheng, Y. Y. Li, and L. H. Yam, "Vibration analysis of annular-like plates," $J$. Sound Vib., 262, No. 5, 1153-1170 (2003).

8. W. M. Lee and J. T. Chen, "Free vibration analysis of a circular plate with multiple circular holes by using indirect BIEM and addition theorem," J. Appl. Mech., 78, No. 1, 011015-1-011015-10 (2011).

9. A. Demir and V. Mermertas, "A study of annular plates with radial through cracks by means of sector type element," J. Sound Vib., 300, No. 3-5, 466-478 (2007).

10. S. Noga and R. Bogacz, "Algorithm to identify the mode shapes of the circular or annular systems with the discontinuous features," in: Proc. of the 20th Int. Conf. on Computer Methods in Mechanics (August 27-31, 2013, Poznań, Poland), Vol. 1, Poznań (2013), pp. MS07-5-6.

11. S. Noga, T. Markowski, and R. Bogacz, "Method of determining the normal modes of toothed gears with complex geometry," Scientific Journal of Silesian University of Technology. Ser. Transport, 89, 119-127 (2015).

12. K. V. Savchenko, A. P. Zinkovskii, I. G. Tokar', and Ya. D. Kruglii, "Influence of the orientation of shroud contact surfaces on the static stress state of turbine rotor blades," Strength Mater., 46, No. 4, 493-502 (2014).

13. S. Wang, J. Xiu, S. Cao, and J. Liu, “Analytical treatment with rigid-elastic vibration of permanent magnet motors with expanding application to cyclically symmetric power-transmission systems," J. Vib. Acoust., 136, No. 2, 021014-1-13 (2014).

14. S. S. Naseralavi, E. Salajegheh, J. Salajegheh, and M. J. Fadaee, "Detection of damage in cyclic structures using an eigenpair sensitivity matrix," Comput. Struct., 110-111, 43-59 (2012).

15. S. Noga, "Transverse vibration analysis of a compound plate with using cyclic symmetry modeling," Vibrations in Physical System, XXVI, 211-216 (2014).

16. P. Nazarko, L. Ziemiański, S. Noga, and T. Markowski, "Comparative analysis of compound annular plates vibration on the basis of numerical and experimental studies," in: Proc. of the 3rd Polish Congress of Mechanics \& 21st Int. Conf. on Computer Methods in Mechanics (September 8-11, 2015, Gdańsk, Poland), Vol. 1, Gdańsk (2015), pp. 421-422. 